\title{
A Novel Surgical Method Using Two Triangular Flaps for Accessory Tragus
}

\author{
Hyun Ho Han ${ }^{1}$, Hak Young Kim², \\ Deuk Young $\mathrm{Oh}^{2}$ \\ ${ }^{1}$ Department of Plastic and \\ Reconstructive Surgery, Incheon St. \\ Mary's Hospital, College of Medicine, \\ The Catholic University of Korea, Seoul; \\ ${ }^{2}$ Department of Plastic and \\ Reconstructive Surgery, Seoul St. Mary's \\ Hospital, College of Medicine, The \\ Catholic University of Korea, Seoul, Korea
}

This study received the Institutional Review Board (IRB) approval from the Catholic Medical Center Office of Human Research Protection Program.

No potential conflict of interest relevant to this article was reported.
Background Accessory tragus is an abnormal ear structure that has the shape of a nodule or a papule. The existing surgical method is very simple, wherein an elliptical incision is made around the lesion and the underlying cartilage is removed. However, this method may leave a depressed or dimpled scar and may cause chondrodermatitis.

Methods We corrected the accessory tragus by a new method using triangular flaps, and the procedure was performed in eight patients. Out of the four triangular flaps, which were created by drawing lines that connected the left and the right sides and the superior and inferior surfaces of the accessory tragus and quartering them, two flaps that faced each other were excised. Once the cartilaginous tissue inside was verified and removed up to the base to the greatest extent possible, the remaining two triangular flaps were sutured and the remaining skin margin of the flap was trimmed.

Results None of the patients had any wound problems related to the surgery, and none of them complained of chondrodermatitis up to 6 months postoperatively. No depression or dimple was observed externally up to 6 months after the operation, and the $z$-shaped scar, which was created by the crossing over of the triangular flaps, was not as visible as a linear scar.

Conclusions Correction of an accessory tragus by using the triangular flaps introduced in this study provides a wider operative view through a smaller incision, while preventing the formation of dimples or a depression after the operation.

Keywords Branchial region, Congenital abnormalities, Surgical flaps

\section{INTRODUCTION}

Accessory tragus is an abnormal ear structure that has the shape of a nodule or a papule [1]. It is generally found right in front of the normal tragus or the crus of the helix. It could be unilateral or bilateral, and may occur with or without a stalk [2]. Histologically, it mostly consists of mature adipose tissue, with sebaceous glands that exist along with eccrine glands and hair follicles, and an elastic

Received: May 24, 2016 Revised: Jun 19, 2016 Accepted: Jun 21, 2016 Correspondence: Deuk Young Oh Department of Plastic and Reconstructive Surgery, Seoul St. Mary's Hospital, College of Medicine, The Catholic University of Korea, 222 Banpo-daero, Seocho-gu, Seoul 16591, Korea. E-mail: ohdeuk1234@hanmail.net

Copyright @ 2016 The Korean Society for Aesthetic Plastic Surgery.

This is an Open Access article distributed under the terms of the Creative Commons Attribution Non-Commercial License (http://creativecommons.org/licenses/by-nc/4.0/) which permits unrestricted non-commercial use, distribution, and reproduction in any medium, provided the original work is properly cited. www.e-aaps.org cartilage is located at the center [3,4]. In general, it does not cause any other accompanying malformation and is mostly a minor congenital malformation. Procedures are performed for resolving aesthetic issues, and pediatric patients are brought to the hospital by their parents.

Various terms are used by physicians to describe an accessory tragus, including supernumerary pinna, supernumerary tragus, rudimentary ear, skin papillus, polyotia, accessory external ear, heterotopic tragus, accessory auricle, and preauricular ear tag, thus creating confusion about the term $[5,6]$. However, since the tragus originates from the first branchial arch, supernumerary tragus or heterotopic tragus is the most accurate term from an embryological aspect [5].

The operation is simple; hence, there is no severe difficulty in performing the procedure or dissent regarding the procedure. In general, an elliptical incision is made around the lesion and the underlying cartilage is removed [1]. The important part of the operation is the removal of the cartilage, because the removal of the base 
of a deep-seated cartilage may lead to the formation of a depression or a dimple in the incision area. Further, shaving the cartilage roughly or leaving behind some of the cartilage may cause wound problems such as chondritis or chondrodermatitis [1].
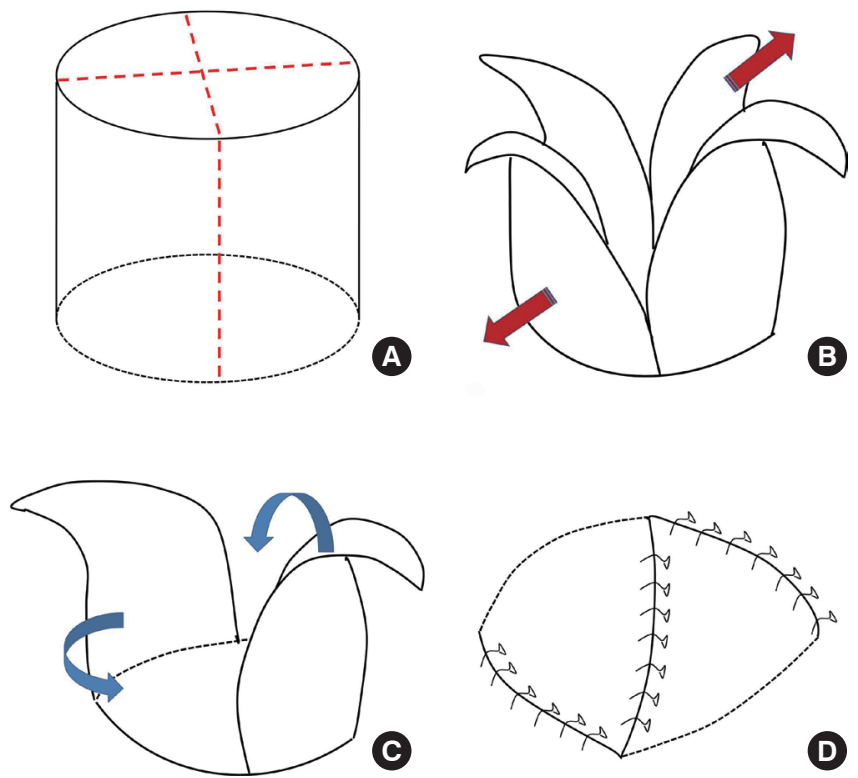

Fig. 1. Surgical technique. (A) A cross was drawn from the accessory tragus using two lines with a large diameter; these lines were quartered to create four triangular flaps. $(B, C)$ After the incision, thick flaps were lifted, and the two facing flaps out of the four triangular flaps were excised. (D) The cartilage tissue was removed from the center to the base; the two remaining triangular flaps were crossed over to close the opening, and the remaining skin margin was trimmed.
In this study, the surgery was performed by using triangular flaps to widen the operative view without extending the length of the incision, while preventing the formation of a skin dimple or depression below the incision. The specific surgical procedure and the results are discussed accordingly.

\section{METHODS}

Our institutional review board approved this study. Eight patients were enrolled between March 2014 and December 2015 on the basis of a retrospective chart review.

\section{Surgical procedure}

In adult patients, the surgery was performed under local anesthesia, and in pediatric patients, it was performed under general anesthesia. A cross was drawn from the accessory tragus using two lines with a large diameter; these lines were quartered to create four triangular flaps (Fig. 1A). After the incision, the flaps were lifted, and the two flaps facing each other were excised (Fig. 1B and 1C). The adipose tissue in the subcutis of the skin flap was sufficiently thick to prevent any circulatory problems in the skin flaps. Once the skin hook was positioned on both flaps when the flaps were lifted, the cartilaginous tissue inside became visible. It was removed up to the base by shaving so that the remaining part was smooth and had no sharp edges. Then, the two remaining triangular flaps were crossed over and sutured, and the skin margin of the flaps was trimmed (Fig. 1D).

In case of an accessory tragus with an existing stalk, the part after the stalk was removed first, and the stalk part was designed as
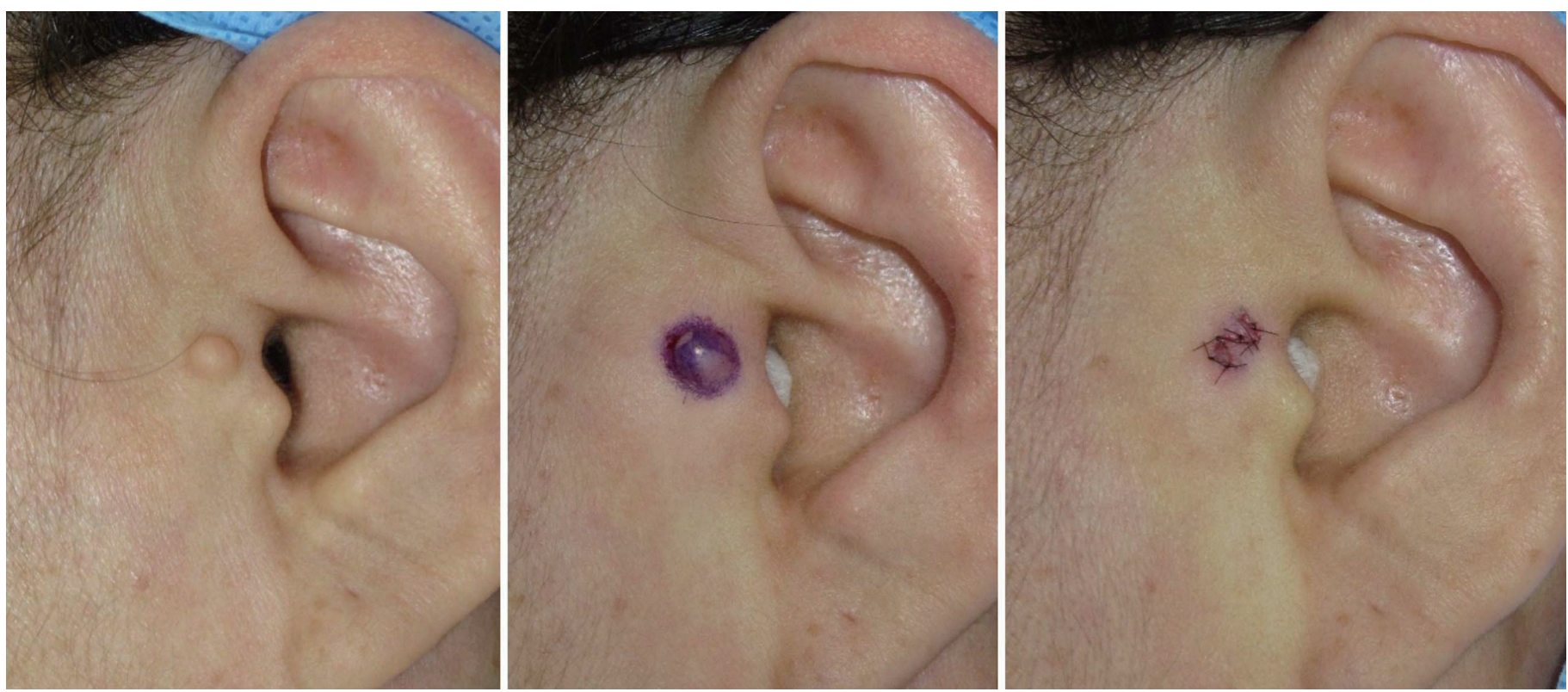

Fig. 2. A 53-year-old female patient: after the operation, the triangular flap was lifted from the skin tissue on the accessory tragus and coverage was achieved. 

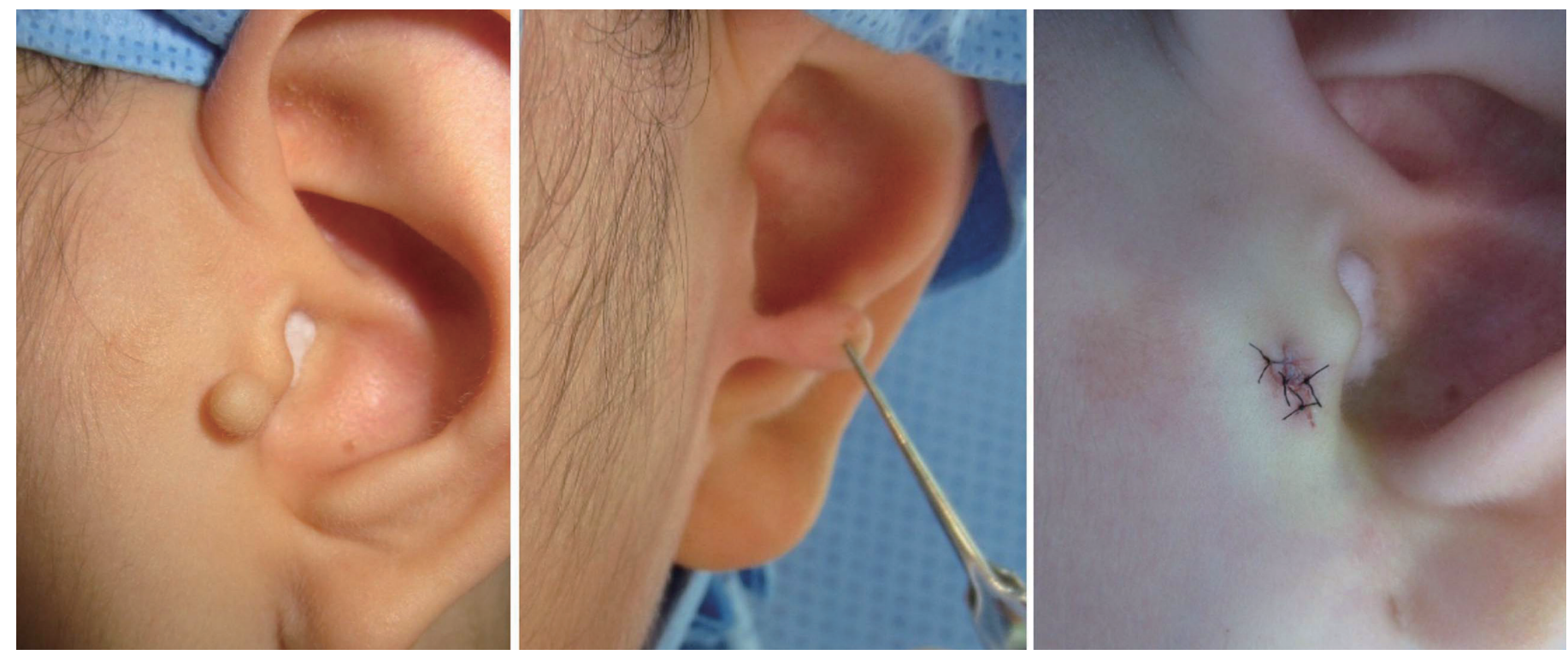

Fig. 3. An 8-year-old female patient with an accessory tragus with a stalk: The stalk was removed and the triangular flap was created with the remaining part.

mentioned above. Then, the operation was performed.

\section{Postoperative care and assessment}

Adult patients who underwent the operation under local anesthesia on an out-patient basis were discharged on the same day, and pediatric patients who underwent the operation under general anesthesia were discharged the day after the operation. Stitches were removed 5 days after the operation. Tapes were applied for 2 weeks after the removal of the stitches, followed by a silicone ointment application for the scar for 3 to 4 months, and progress was observed at 6 months after the operation. Immediate problems directly related to the operation, such as necrosis, wound disruption, hemato$\mathrm{ma}$, or infection of the flap, were verified, along with the occurrence of chondrodermatitis.

\section{RESULTS}

Eight patients underwent the operation (Fig. 2). The patient group included three adults and five children, with an average age of 20 years, and the age range was from 24 months to 59 years. The average follow-up period was 6.3 months. There was no case of bilateral accessory tragus, but all cases had unilateral accessory tragus, and two out of the eight patients had multiple accessory tragi, with two accessory tragi on one side. No accompanying congenital anomaly was detected, and no familial type was observed. A stalk was present in one patient (Fig. 3).

After the surgery, no operation-related wound problems, such as necrosis, wound disruption, hematoma, and infection, occurred, and none of the patients complained of chondrodermatitis up to 6 months after the operation. Externally, no depression or dimple was observed during the 6-month follow-up period, and the z-shaped scar that was formed by the crossing over of the triangular flaps was not as visible as a linear scar (Fig. 4).

\section{DISCUSSION}

The congenital condition of accessory tragus was reported in 1858 by Birkett [7]. Its prevalence rate in unilateral cases is 1.7:1,000 and in bilateral cases is 9-10:10,000 [8]. It is a very rare disease with a lower prevalence rate than the prevalence rate of 1.9-2.2:1,000 for cleft lip and palate [9]. Accessory tragus is usually 3 to $10 \mathrm{~mm}$ in size, appearing in the shape of a nodule or a papule, and mostly requires treatment for aesthetic reasons.

Since the procedure is simple and there is no objection to it, there are almost no reports on the technical aspects of this operation in the literature. However, the existing method, which consists of an elliptical incision, cartilage shaving, and closure, leaves a conspicuous linear scar in the incision area and may cause a dimple or depression, while unsmooth shaving of the cartilage may continuously cause chondrodermatitis.

The operative design in this study includes the utilization of the skin over the accessory tragus as flaps. Less tissue is wasted than with the use of the elliptical design, and the incision is shorter (Fig. 5). Further, securing a view through the triangular flaps is easier, and access to the deep base attachment of the cartilage is also easy. As the triangular flaps are closed in a crossover manner, the possibility of developing a depression or of the dimpling of the scar is reduced. As previously mentioned, the cartilage should be completely removed or smoothly shaven to prevent the development of chondrodermatitis in the future, and since the triangular skin flap 

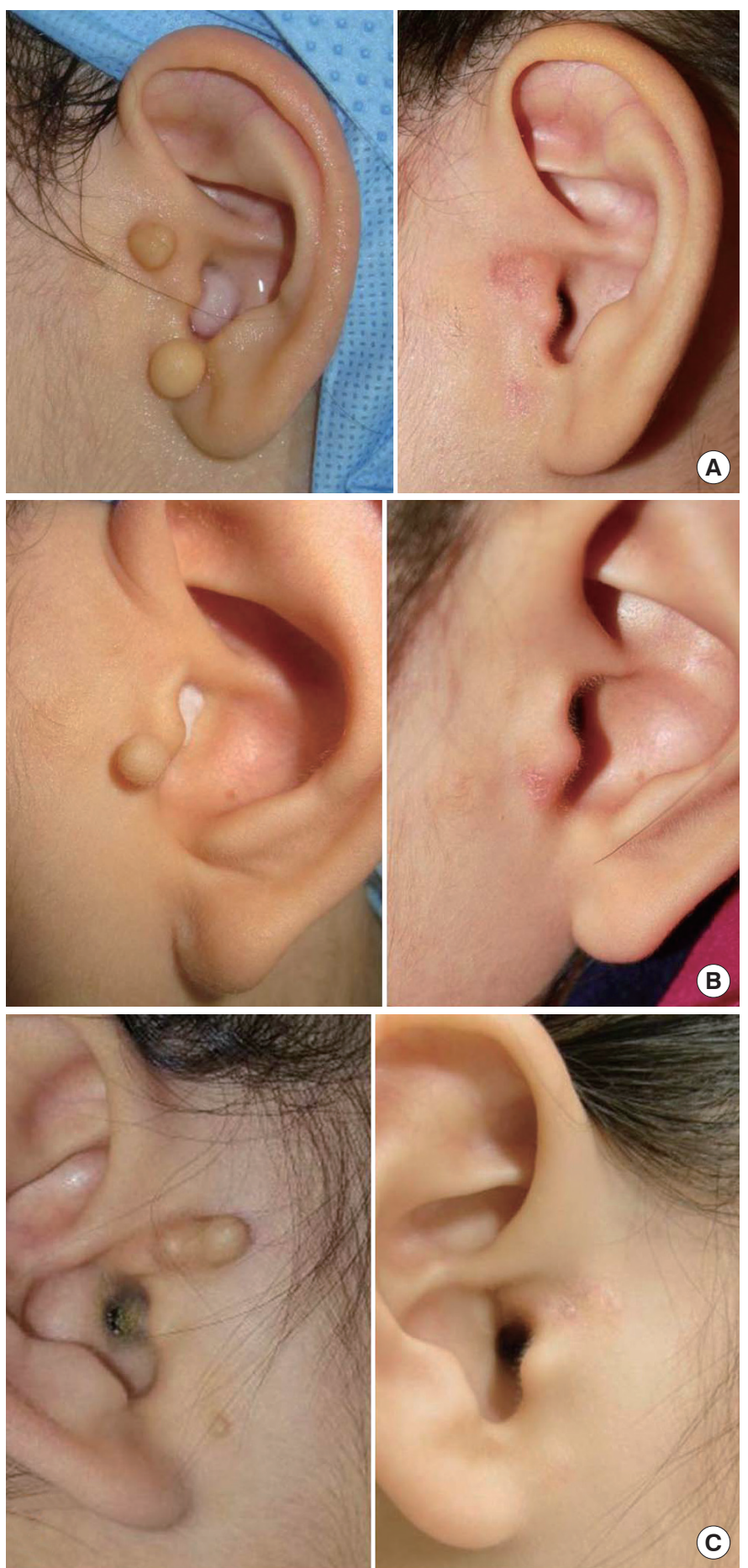

Fig. 4. Postoperative results. No surgery-related wound problems, such as necrosis, wound disruption, hematoma, and infection, were observed in the flap after the operation, and the z-shaped scar, which is formed as the triangular flaps cross over, was less visible than the linear scar.

cross-covers the cartilage, it provides a favorable point from this perspective as well.

Before performing a surgical treatment of an accessory tragus,

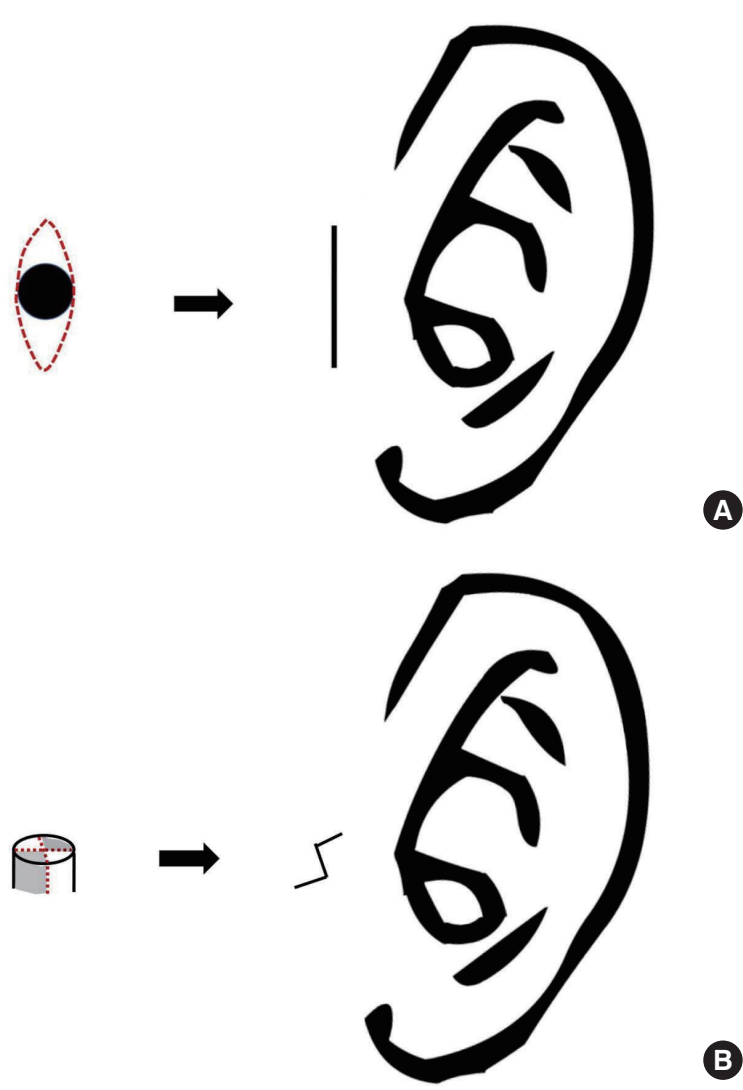

Fig. 5. The operation design (B) in this study utilizes the skin over the accessory tragus as the flap. A smaller volume of tissue is wasted than with use of the elliptical design (A), and the incision is shorter.

any accompanying congenital anomaly should be identified. Embryologically, the auricular region is formed from the first and the second branchial arches, and the six hillocks of His (mesenchymal tubercles) are formed from these two arches at the end of the fifth week of embryonic life; they combine to form the entire auricular structure [10-12]. Among them, the first hillock forms the tragus, and a problem is suspected in this process when an accessory tragus develops; therefore, verification of any malformation related to the first and the second branchial arches is required.

Syndromic malformations related to the first and the second branchial arches include accessory tragus, along with epibulbar dermoid cyst, oculo-auriculo-vertebral syndrome (Goldenhar syndrome) that has defects in the vertebral column, and hemifacial macrosomia that is frequently accompanied with an auricular defect [13]. Although very rare, mandibulofacial dysostosis (Treacher Collins syndrome), Townes-Brocks syndrome, VACTERL syndrome, and $4 p$ syndrome (Wolf-Hirschhorn syndrome) might be accompanied by accessory tragus [14]. Further, an increased possibility of problems in auditory perception among pediatric patients with accessory tragus has been reported; hence, a hearing test should be performed in all patients [2].

In conclusion, accessory tragus is a minor congenital malforma- 
tion, and if it is corrected using the triangular flap design introduced in this study, a wider view with a smaller incision could be obtained, the formation of dimples and depression after the operation could be prevented, and a less visible scar would be left.

\section{PATIENT CONSENT}

Patients provided written consent for the use of their images.

\section{REFERENCES}

1. Sebben JE. The accessory tragus--no ordinary skin tag. J Dermatol Surg Oncol 1989;15:304-7.

2. Jansen T, Romiti R, Altmeyer P. Accessory tragus: report of two cases and review of the literature. Pediatr Dermatol 2000;17:391-4.

3. Satoh T, Tokura Y, Katsumata M, et al. Histological diagnostic criteria for accessory tragi. J Cutan Pathol 1990;17:206-10.

4. Cosman BC. Bilateral accessory tragus. Cutis 1993;51:199-200.

5. Bahrani B, Khachemoune A. Review of accessory tragus with highlights of its associated syndromes. Int J Dermatol 2014;53:1442-6.
6. Miller CS, Miller KF. Supernumerary ears; report of three cases. Arch Derm Syphilol 1949;60:601-8.

7. Shin MS, Choi YJ, Lee JY, et al. A case of accessory tragus on the nasal vestibule. Ann Dermatol 2010;22:61-2.

8. Melnick M, Myrianthopoulos NC, Paul NW. External ear malformations: epidemiology, genetics, and natural history. Birth Defects Orig Artic Ser 1979;15:i-ix, 1-140.

9. Han HH, Choi EJ, Kim JM, et al. The importance of multidisciplinary management during prenatal care for cleft lip and palate. Arch Plast Surg 2016;43:153-9.

10. Wood-Jones F, W IC. The development of the external ear. J Anat 1934;68:525-33.

11. Mc KJ. The first arch syndrome. Arch Dis Child 1958;33:477-86.

12. Williams GH. Developmental anatomy of the ear. In: English GM, editor. Otolaryngology. Philadelphia, PA: JB Lippincott; 1994. p.1-13.

13. Stoll C, Viville B, Treisser A, et al. A family with dominant oculoauriculovertebral spectrum. Am J Med Genet 1998;78:345-9.

14. Resnick KI, Soltani K, Bernstein JE, et al. Accessory tragi and associated syndromes involving the first branchial arch. J Dermatol Surg Oncol 1981;7:39-41. 